\title{
SUSCETIBILIDADE DE POPULAÇÕES DE Brevipalpus phoenicis (GEIJSKES, 1939) (ACARI: TENUIPALPIDAE) A ACARICIDAS ORGANOESTÂNICOS EM CITROS'
}

\author{
Roberto Hiroyuki Konno ${ }^{2,5}$; Claudio Roberto Franco ${ }^{3,6}$; Celso Omoto4* \\ ${ }^{2}$ Pós-Graduando em Entomologia - USP/ESALQ. \\ ${ }_{4}^{3}$ Graduando em Engenharia Agronômica - USP/ESALQ. \\ ${ }^{4}$ Depto. de Entomologia, Fitopatologia e Zoologia Agrícola - USP/ESALQ, C.P. 9 - CEP: 13418-900 - Piracicaba, SP. \\ ${ }^{5}$ Bolsista FAPESP. \\ ${ }^{6}$ Bolsista PIBIC/CNPq. \\ *Autor correspondente <celomoto@carpa.ciagri.usp.br>
}

RESUMO: Brevipalpus phoenicis (Geijskes, 1939) é uma das principais pragas da cultura de citros no Brasil. Os acaricidas organoestânicos representam mais $40 \%$ da área tratada de citros para o controle de $B$. phoenicis. O objetivo do presente trabalho foi o de coletar informações básicas para a implementação de um programa de manejo da resistência de $B$. phoenicis a acaricidas organoestânicos em pomares de citros do Estado de São Paulo preservando, dessa forma, a vida útil dos acaricidas deste grupo químico. Inicialmente, as linhas básicas de suscetibilidade aos acaricidas óxido de fenbutatin e cihexatin foram obtidas para uma população suscetível de referência de $B$. phoenicis através de um bioensaio de contato residual. Baseado na curva de concentração-resposta dessa população para os dois acaricidas, a concentração diagnóstica de $180 \mathrm{mg}$ de $[\mathrm{IA}] \mathrm{L}^{-1}$ de água destilada foi definida para ser utilizada em programas de monitoramento da suscetibilidade de populações de $B$. phoenicis a esses acaricidas. Posteriormente, foi realizado um levantamento da suscetibilidade aos acaricidas óxido de fenbutatin e cihexatin em 26 populações de $B$. phoenicis coletadas em pomares comerciais com diferentes regimes de uso de acaricidas organoestânicos nos últimos cinco anos. Todas as populações apresentaram suscetibilidade a óxido de fenbutatin e cihexatin semelhante ao da população suscetível de referência, com exceção de uma população que apresentou uma porcentagem de sobrevivência a cihexatin $(10,7 \%)$ significativamente maior do que a da população suscetível $(1,92 \%)$ na concentração diagnóstica. Portanto, apesar do intenso uso de acaricidas organoestânicos para controle de $B$. phoenicis em citros no Estado de São Paulo, as populações desse ácaro ainda apresentam uma alta suscetibilidade aos acaricidas óxido de fenbutatin e cihexatin.

Palavras-chave: Citrus, óxido de fenbutatin, cihexatin, resitência, pesticidas

\section{SUSCEPTIBILITY OF Brevipalpus phoenicis (GEIJSKES, 1939) (ACARI: TENUIPALPIDAE) TO ORGANOTIN ACARICIDES IN CITRUS}

\begin{abstract}
Brevipalpus phoenicis (Geijskes, 1939) is one of the most important citrus pests in Brazil. Organotin acaricides represent more than $40 \%$ of the acaricide-treated citrus area for controlling B. phoenicis. For preserving the life-time of organotin acaricides, the objective of this research was to collect basic information for implementing a resistance management program of $B$. phoenicis to organotin acaricides in citrus groves from the State of São Paulo, Brazil. Initially, baseline susceptibility data of the susceptible reference population of $B$. phoenicis to fenbutatin oxide and cyhexatin were obtained with the use of a residual contact bioassay. Based on concentration-response data of this population to these acaricides, a diagnostic concentration of $180 \mathrm{mg}$ of [Al] $\mathrm{L}^{-1}$ of distilled water was defined for both fenbutatin oxide and cyhexatin in order to be used in a program for monitoring the susceptibility of $B$. phoenicis to these acaricides. Then, a survey of the susceptibility to fenbutatin oxide and cyhexatin was conducted in 26 populations of $B$. phoenicis collected from citrus groves with different regimes of organotin acaricide use in the past five years. All populations showed susceptibilities to fenbutatin oxide and cyhexatin similar to the susceptible population; except for one population which showed a significant higher survivorship to cyhexatin $(10,7 \%)$ than that for the susceptible population $(1,92 \%)$ at the diagnostic concentration. Therefore, although the intense use of organotin acaricides in citrus groves from the State of São Paulo, the susceptibilities of $B$. phoenicis populations to fenbutatin oxide and cyhexatin are still very high.

Key words: Citrus, fenbutatin oxide, cyhebatin, resistance, pesticide
\end{abstract}

\section{INTRODUÇÃO}

O ácaro da leprose Brevipalpus phoenicis (Geijskes, 1939) (Acari: Tenuipalpidae) é uma das principais pragas da cultura do citros no Brasil devido aos sérios prejuízos que acarreta na produção, principalmente, em virtude da transmissão do agente causal da leprose dos citros (Oliveira, 1986; Chiavegato, 1987; Kitajima et al., 1995). 
$O$ controle de $B$. phoenicis tem sido realizado, principalmente, através da utilização de produtos químicos. Estima-se que 90 milhões de dólares são gastos com acaricidas na citricultura brasileira, o que significa, aproximadamente, $20 \%$ do custo de produção (Salvo Filho, 1997). Segundo o levantamento apresentado por Nakano (1995), 44\% dos ingredientes ativos comercializados no ano de 1994, para o controle de $B$. phoenicis, pertenciam ao grupo dos organoestânicos (por ex.: óxido de fenbutatin, cihexatin e azociclotin). Aproximadamente $40 \%$ da área tratada com acaricidas em citros no Brasil são com os organoestânicos. Atualmente este grupo de acaricidas ainda tem sido o mais utilizado devido a sua eficácia no controle de $B$. phoenicis, associada a uma certa seletividade aos inimigos naturais (Yamamoto, 1995).

Apesar do grande esforço direcionado ao controle de $B$. phoenicis, problemas relacionados à eficiência de controle com alguns acaricidas têm sido esporadicamente reportados por agricultores e técnicos ligados à área citrícola. Devido à intensa aplicação de acaricidas, pode-se levantar a hipótese de que a evolução da resistência de $B$. phoenicis a acaricidas seja um dos fatores que vem contribuindo para a redução da eficácia de alguns acaricidas (Omoto, 1995a, b; 1998).

Em outros países, vários estudos têm relacionado falhas no controle de ácaros da família Tetranychidae com a utilização de acaricidas organoestânicos ao problema da resistência. $\mathrm{Na}$ Austrália, os casos de resistência têm sido relacionados principalmente em Tetranychus urticae Koch aos acaricidas cihexatin e óxido de fenbutatin (Edge \& James, 1986; Herron et al., 1994; Goodwin et al., 1995). Nos Estados Unidos, os casos de resistência aos acaricidas organoestânicos têm sido relatados em $T$. urticae e Panonycus ulmi Koch em fruteiras (Hoyt et al., 1985; Keena \& Granett, 1987; Welty et al., 1987; Hoy et al., 1988; Tian et al., 1992). Casos de resistência de ácaros aos acaricidas organoestânicos também têm sido relatados no Canadá, com detecção de populações de $P$. ulmi resistentes ao cihexatin (Pree \& Wagner, 1987) e no Reino Unido, onde Jacobson et al. (1999) relataram a resistência ao óxido de fenbutatin em populações de de $T$. urticae coletadas em roseiras.

Com a finalidade de coletar informações básicas para implementação de um programa de manejo da resistência do ácaro da leprose aos acaricidas óxido de fenbutatin e cihexatin, os objetivos do presente trabalho foram: caracterizar a linha básica de suscetibilidade de B. phoenicis aos acaricidas óxido de fenbutatin e cihexatin; avaliar o efeito de diferentes regimes de uso de acaricidas organoestânicos sobre a suscetibilidade de populações de $B$. phoenicis aos acaricidas óxido de fenbutatin e cihexatin.

\section{MATERIAL E MÉTODOS}

O presente projeto de pesquisa foi desenvolvido no Laboratório de Resistência de Artrópodes a Pesticidas do Departamento de Entomologia, Fitopatologia e Zoologia Agrícola da Escola Superior de Agricultura "Luiz de Queiroz", Universidade de São Paulo, Piracicaba - SP, no período de março de 1998 a abril de 2000 .

\section{Criação de Brevipalpus phoenicis em laboratório}

Os ácaros foram criados em frutos de laranja das variedades Pera Rio ou Valência. Os frutos foram lavados em água corrente, secados e mergulhados em parafina derretida, deixando-se uma área de aproximadamente $10 \mathrm{~cm}^{2}$ para a colonização dos ácaros. Esta área foi circundada com uma barreira com cola adesiva (marca Tanglefoot ${ }^{\circledR}$ ) para confinar os ácaros. Antes da transferência dos ácaros, uma mistura de gesso, areia, farinha de trigo e água foi pincelada em alguns pontos da arena, simulando os sintomas da verrugose, os quais favorecem o desenvolvimento de $B$. phoenicis (Chiavegato, 1986 e Nakano et al., 1987). Cada fruto recebeu de 50 a 100 ácaros fêmeas, totalizando 24 a 36 frutos por população, mantidos em sala climatizada à temperatura de $25 \pm 1^{\circ} \mathrm{C}$, umidade relativa de $70 \pm 10 \%$ e fotofase de $14 \mathrm{~h}$. A renovação dos frutos foi realizada mensalmente quando um fruto novo era colocado sobre o fruto velho para que ocorresse a transferência dos ácaros.

\section{Método de bioensaio}

As respostas das populações de $B$. phoenicis aos acaricidas organoestânicos óxido de fenbutatin e cihexatin foram avaliadas através de um bioensaio residual.

As diversas concentrações de óxido de fenbutatin (Torque $500 \mathrm{SC}^{\circledR}, 500 \mathrm{~g}$ de óxido de fenbutatin $\mathrm{L}^{-1}$ de produto - Cyanamid Química do Brasil Ltda.) e cihexatin (Hokko Cyhexatin $500^{\circledR}$, pó molhável, $500 \mathrm{~g}$ de cihexatin $\mathrm{kg}^{-1}$ de produto - Hokko do Brasil Ltda) foram preparadas a partir da diluição dos produtos comerciais em água destilada. Discos de folha de laranjeira de 2,5 $\mathrm{cm}$ de diâmetro, obtidos por meio de um vazador, foram pulverizados individualmente na superfície adaxial, com o auxílio da torre de Potter (Burkard Manufacturing, Rickmansworth, Inglaterra), calibrada a uma pressão de $68,95 \mathrm{kPa}$ (10 psi). Um volume de $2 \mathrm{~mL}$ de solução foi utilizada na pulverização de modo a se obter uma deposição média de resíduo úmido de $1,56 \mathrm{mg} \mathrm{cm}^{-2}$. Uma vez secos, os discos foram transferidos para placas de petri de $3,5 \mathrm{~cm}$ de diâmetro contendo uma camada de mistura não geleificada de ágar água a 3,0\%, no fundo. Com essa mesma mistura foi feita uma barreira na borda do disco para evitar a fuga dos ácaros (adaptado de Vestergaard et al., 1995). Para cada arena foram transferidos 10 ácaros fêmeas adultos, com o auxílio de um pincel de pelos finos e microscópio estereoscópico. Após a transferência, as placas foram tampadas e 
mantidas em câmara climatizada à temperatura de $25 \pm$ $1^{\circ} \mathrm{C}$ e fotofase de $14 \mathrm{~h}$. A mortalidade foi avaliada $24 \mathrm{~h}$ após a transferência dos ácaros. O critério de mortalidade foi baseado no toque de cada ácaro com o auxílio de um pincel. Os indivíduos que não responderam com movimentos vigorosos de seus apêndices foram considerados mortos. Em cada bioensaio, as repetições que apresentaram uma mortalidade superior a $10 \%$ na testemunha foram descartadas.

\section{Caracterização toxicológica de populações de Brevipalpus phoenicis a óxido de fenbutatin e cihexatin}

Três populações de $B$. phoenicis foram escolhidas para a caracterização toxicológica a óxido de fenbutatin e cihexatin. Uma população de laboratório, mantida na ausência de pressão de seleção com acaricidas desde 1994, foi tomada como a população susceptível de referência (S). Outra população, denominada de Col-1, oriunda de um pomar comercial do Município de Colômbia-SP, onde o uso de acaricidas organoestânicos foi bastante intenso e uma terceira população, denominada de Pir-1, proveniente de um pomar não comercial do Município de Piracicaba-SP.

Para as caracterizações toxicológicas das populações aos dois produtos, foram utilizadas cinco concentrações espaçadas logaritmicamente entre 18 a $180 \mathrm{mg}$ de óxido de fenbutatin ou cihexatin $\mathrm{L}^{-1}$ de água destilada. Os bioensaios foram repetidos de 3 a 8 vezes para cada concentração do produto ao longo do tempo.

Os dados de mortalidade obtidos para cada população e produtos foram submetidos à análise de Próbite (LeOra Software, 1987). Baseado na resposta de concentração-mortalidade, uma concentração diagnóstica foi escolhida para óxido de fenbutatin e cihexatin seguindo os critérios apresentados por Roush \& Miller (1986) e FFrench-Constant \& Roush (1990).

\section{Monitoramento da suscetibilidade de populações de $B$. phoenicis aos acaricidas óxido de fenbutatin e cihexatin}

No primeiro semestre de 1999 foram coletadas populações de $B$. phoenicis provenientes de diferentes pomares comerciais de citros do Estado de São Paulo, onde o histórico de uso de acaricidas tem sido registrado nos últimos anos. Dessa forma, foi possível identificar em um determinado pomar, talhões que receberam diferentes regimes de uso de acaricidas organoestânicos (TABELA 1). De cada talhão foram coletados 30 a 50 frutos infestados com ácaros, sendo estes estabelecidos em condições de laboratório. Essas populações foram denominadas de Fischer-1, Fischer-2, ... e Fischer-26 uma vez que foram provenientes de talhões das Fazendas de propriedade da Fischer Agropecuária S.A.

Com base na caracterização toxicológica da população $S$ ao óxido de fenbutatin e cihexatin, a concentração de $180 \mathrm{mg} \mathrm{L}^{-1}$ para ambos os produtos foi selecionada como a concentração diagnóstica para o monitoramento da suscetibilidade de populações de $B$. phoenicis a esses acaricidas. No monitoramento com óxido de fenbutatin, as diferentes populações testadas foram submetidas a oito repetições ao longo do tempo e em cada repetição foram utilizados aproximadamente 50 ácaros. No monitoramento com cihexatin, as populações avaliadas foram submetidas a cinco repetições ao longo do tempo, sendo que em cada repetição foram testados aproximadamente 40 ácaros. Os dados de porcentagem de sobrevivência $(X)$ das populações de cada monitoramento foram transformados em arc sen $\sqrt{(X / 100)}$ e as médias, comparadas pelo teste bilateral de Dunnett a $5 \%$, tendo como referência a sobrevivência obtida pela população $S$.

\section{RESULTADOS E DISCUSSÃO}

\section{Caracterização toxicológica de populações de Brevipalpus phoenicis a óxido de fenbutatin e cihexatin}

Não foram observadas diferenças significativas em termos de resposta das populações $S$, Col-1 e Pir-1 tanto para o acaricida óxido de fenbutatin (TABELA 2) com para o cihexatin ( TABELA 3).

Para a caracterização toxicológica ao óxido de fenbutatin (TABELA 2) foram testados 1760,882 e 816 ácaros para as populações $S$, Col-1 e Pir-1, respectivamente. Para a população $\mathrm{S}$, a $\mathrm{CL}_{50}$ estimada foi de $59,33 \mathrm{mg} \mathrm{L}^{-1}$ de óxido de fenbutatin (I.C. $95 \%$ $55,87-63,01)$ e coeficiente angular ( \pm desvio padrão) de $2,76( \pm 0,12), \chi^{2}$ de 2,30 (graus de liberdade (g.l.) = 3; $P>0,05)$. $A \mathrm{CL}_{50}$ estimada para a população Col-1 foi de $85,06 \mathrm{mg} \mathrm{L}^{-1}$ de óxido de fenbutatin (I.C. 95\% 61,42$134,11)$ e coeficiente angular de $3,21( \pm 0,19), \chi^{2}$ de 8,45 (g $\left.\mathrm{L}^{-1}=3 ; P>0,05\right)$. Já, para a população Pir-1, $\mathrm{CL}_{50}$ estimada foi de $74,33 \mathrm{mg} \mathrm{L}^{-1}$ de óxido de fenbutatin (I.C. $95 \% 60,91-85,96)$ e coeficiente angular de 3,47 $( \pm 0,29)$, $\chi^{2}$ de 2,62 (g.l. $=3 ; P>0,05$ ).

Observando as $\mathrm{CL}_{50} \mathrm{~s}$ estimadas de óxido de fenbutatin das três populações, verifica-se uma sobreposição dos intervalos de confiança. Essa sobreposição indica que as $\mathrm{CL}_{50} \mathrm{~s}$ estimadas não diferem entre si. Dado semelhante foi obtido por Alves (1999) que realizou a caracterização toxicológica de uma população de $B$. phoenicis ao óxido de fenbutatin, obtendo uma $\mathrm{CL}_{50}$ estimada de aproximadamente 67 $\mathrm{mg} \mathrm{L}^{-1}$. Portanto, apesar da população Col-1 ter recebido uma intensa pulverização com acaricidas organoestânicos, não foi verificado uma resposta à pressão de seleção.

Através do teste de paralelismo das linhas de concentração-resposta, obtidas para o óxido de fenbutatin por meio da análise de Próbite ( TABELA 2), verifica-se que a população $S$ apresentou um coeficiente angular significativamente menor que as populações Col1 e Pir-1 $\left(\chi^{2}=6,93 ;\right.$ g.l. $\left.=2 ; P<0,05\right)$. Segundo alguns 
autores, o coeficiente angular relaciona-se à homogeneidade de uma população (Hoskins \& Gordon, 1956) ou ao método de bioensaio utilizado na caraterização toxicológica (FFrench-Constant \& Roush, 1990). A diferença observada não pode estar relacionada ao método de bioensaio, uma vez que a caracterização toxicológica foi realizada através do mesmo método paras as três populações. Os bioensaios realizados com óxido de fenbutatin apresentaram uma quantidade de ácaros moribundos muito elevado, dificultando a distinção entre indivíduos vivos e mortos. Assim, o menor coeficiente angular, apresentado pela população $S$, pode estar associado ao critério de mortalidade utilizado no início do experimento. Um dos grandes problemas envolvendo os trabalhos de detecção e monitoramento da resistência onde se avalia por meio de um bioensaio,

TABELA 1 - Populações de Brevipalpus phoenicis provenientes de pomares comerciais de citros da Fischer Agropecuária S.A. que receberam diferentes regimes de uso de acaricidas organoestânicos no período de 1995-1999.

\begin{tabular}{llllll}
\hline \multirow{2}{*}{ População } & \multicolumn{5}{c}{ Ano de aplicação } \\
\cline { 2 - 6 } & 1995 & 1996 & 1997 & 1998 & 1999 \\
\hline
\end{tabular}

Fischer-1

\begin{tabular}{|c|c|c|c|c|c|}
\hline Fischer-2 & $===^{(1)}$ & & & & \\
\hline Fischer-3 & $====$ & $====$ & & & \\
\hline Fischer-4 & $====$ & $====$ & & & \\
\hline Fischer-5 & $====$ & $====$ & & $====$ & \\
\hline Fischer-6 & $====$ & $====$ & $====$ & & \\
\hline Fischer-7 & $====$ & $====$ & $====$ & & \\
\hline Fischer-8 & $====$ & $====$ & $====$ & & $====$ \\
\hline Fischer-9 & $====$ & & $====$ & & $====$ \\
\hline Fischer-10 & $====$ & $====$ & & & $====$ \\
\hline Fischer-11 & & $====$ & & & \\
\hline Fischer-12 & & $====$ & $====$ & & \\
\hline Fischer-13 & & $====$ & $====$ & & \\
\hline Fischer-14 & & $====$ & $====$ & & \\
\hline Fischer-15 & & $====$ & $====$ & & \\
\hline Fischer-16 & & $====$ & $====$ & & $====$ \\
\hline Fischer-17 & & $====$ & $====$ & & $====$ \\
\hline Fischer-18 & & $====$ & & $====$ & \\
\hline Fischer-19 & & $====$ & & & $====$ \\
\hline Fischer-20 & & & $====$ & & \\
\hline Fischer-21 & & & $====$ & $====$ & \\
\hline Fischer-22 & & & & $====$ & \\
\hline Fischer-23 & & & & $====$ & \\
\hline Fischer-24 & & & & $====$ & $====$ \\
\hline Fischer-25 & & & & & $====$ \\
\hline Fischer-26 & & & & & $====$ \\
\hline
\end{tabular}

(1)Ano em que recebeu pulverização com acaricidas organoestânicos (apenas uma aplicação por ano). a morte ou sobrevivência de um organismo, é a adoção de um critério de mortalidade adequado e a dificuldade em distinguir indivíduos vivos de mortos em bioensaios ocorre com certa freqüência (Hoskins \& Craig, 1962). Welty et al. (1988) salientam a importância do critério de mortalidade na avaliação da suscetibilidade de Panonychus ulmi (Koch) (Acari: Tetranychidae) ao acaricida cihexatin e demonstraram que a melhor discriminação entre indivíduos suscetível e resistente ocorreu quando os ácaros moribundos não foram considerados como mortos. Em bioensaio residual, os ácaros incapazes de se locomover, mas capazes de algum movimento de perna poderiam ser considerados como mortos. A inclusão de ácaros moribundos como mortos, na avaliação de um bioensaio residual, é correto se os ácaros cessarem a alimentação e a oviposição e tão logo morrerem (Welty et al., 1988). Em trabalhos preliminares realizados para o presente estudo, foi verificado que ácaros moribundos não conseguem sobreviver por $72 \mathrm{~h}$ após a infestação. Sendo assim, a avaliação da mortalidade foi realizada $24 \mathrm{~h}$ após a infestação, considerando-se ácaros moribundos (ou seja, os ácaros com movimentos dos apêndices de forma desordenada e incapazes de se locomover) como mortos. Esse critério foi adotado para a obtenção de uma resposta mais rápida nos bioensaios e evitar que ácaros sobreviventes se percam na barreira de ágar após um maior período de contato com o resíduo do produto. Nessas condições, haveria a possibilidade de se subestimar o problema da resistência.

Para a caracterização toxicológica ao acaricida cihexatin (TABELA 3) foram utilizados 977, 662 e 675 ácaros fêmeas adultos para as populações $S$, Col-1 e Pir-1, respectivamente. Para a população $S$, a $C_{5}$ estimada foi de $70,52 \mathrm{mg} \mathrm{L}^{-1}$ de cihexatin (I.C. $95 \%$ $56,30-89,85)$ e coeficiente angular de $3,69( \pm 0,20), \chi^{2}$ de

TABELA 2 - Respostas de concentração-mortalidade das populações S, Col-1 e Pir-1 de Brevipalpus phoenicis ao acaricida óxido de fenbutatin.

\begin{tabular}{lccc}
\hline População & $\mathrm{n}^{*}$ & $\begin{array}{c}\text { CL50 }\left(\mathrm{mg} \mathrm{I.A} \mathrm{L} \mathrm{L}^{-1}\right) \\
(95 \% \text { IC })\end{array}$ & $\begin{array}{c}\text { Coef. Angular } \\
\text { desvio padrão }\end{array}$ \\
\hline S & 1.760 & $59,33(55,87-63,01)$ & $2,76 \pm 0,12$ \\
Col-1 & 882 & $85,06(61,42-134,11)$ & $3,21 \pm 0,19$ \\
Pir-1 & 816 & $74,33(60,91-85,96)$ & $3,47 \pm 0,29$ \\
\hline
\end{tabular}

*Número de ácaros testados

TABELA 3 - Respostas de concentração-mortalidade das populações S, Col-1 e Pir-1 de Brevipalpus phoenicis ao acaricida cihexatin.

\begin{tabular}{lccc}
\hline População & $\mathrm{n}^{*}$ & $\begin{array}{c}\text { CL50 (mg l.A L-1) } \\
(95 \% \text { IC })\end{array}$ & $\begin{array}{c}\text { Coef. Angular } \pm \\
\text { desvio padrão }\end{array}$ \\
\hline S & 977 & $70,52(56,30-89,85)$ & $3,69 \pm 0,20$ \\
Col-1 & 662 & $68,01(54,21-86,93)$ & $3,95 \pm 0,26$ \\
Pir-1 & 675 & $70,31(60,68-81,98)$ & $3,99 \pm 0,26$ \\
\hline
\end{tabular}

${ }^{*}$ Número de ácaros testados 
$12,21$ (g.l. $=3 ; P>0,05) . A C_{50}$ estimada para a população Col-1 foi de $68,01 \mathrm{mg} \mathrm{L}^{-1}$ de cihexatin (I.C. $95 \% 54,21-86,93)$ e coeficiente angular de 3,95 $( \pm 0,26)$, $\chi^{2}$ de 8,95 (g.l. $=3 ; P>0,05$ ). Já, para a população Pir-1, $\mathrm{CL}_{50}$ estimada foi de $70,31 \mathrm{mg} \mathrm{L}^{-1}$ de cihexatin (I.C. $95 \%$ $60,68-81,98)$ e coeficiente angular de $3,99( \pm 0,26), \chi^{2}$ de 4,06 (g.l. $=3 ; P>0,05$ ).

Observando as $\mathrm{CL}_{50} \mathrm{~s}$ estimadas das três populações ao cihexatin, verifica-se uma sobreposição dos intervalos de confiança. Essa sobreposição indica que as $\mathrm{CL}_{50}$ s estimadas, para a população S, Col-1 e Pir1 não diferem entre si, evidenciando que a intensidade de uso de acaricidas organoestânicos não interferiu na mudança da $\mathrm{CL}_{50}$.

Por meio do teste de paralelismo das linhas de regressão estimada pela análise de Próbite, observouse que a resposta das três populações foram semelhantes ao acaricida cihexatin $\left(\chi^{2}=1,07\right.$; g.l. $=2$; $P>0,05)$ ( TABELA 3).

Baseado na linha de concentração-resposta da população $S$ ao óxido de fenbutatin (Figura 1A) e cihexatin (Figura 1B) a concentração de $180 \mathrm{mg} \mathrm{L}^{-1}$ foi definida para os dois produtos como uma concentração diagnóstica a ser utilizada em programas de monitoramento da suscetibilidade de populações de $B$. phoenicis a esses acaricidas. Nessa concentração, a mortalidade da população $S$ é de aproximadamente 95\% tanto para óxido de fenbutatin como para cihexatin.

Os testes toxicológicos mostraram que as três populações responderam de forma semelhante aos acaricidas óxido de fenbutatin e cihexatin. Esses resultados podem ser em função dos testes terem sido realizados em condições de laboratório onde, geralmente, há uma maior uniformidade de pulverização e um contato bastante intenso entre a praga e o produto, não havendo possibilidade dos ácaros escaparem para locais não tratados. Portanto, o bioensaio utilizado em laboratório pode não ser suficientemente acurado a ponto de discriminar os indivíduos resistentes, subestimando o problema da resistência. Em condições de campo, a pulverização é desuniforme, sendo que o contato entre a praga e o produto ocorre de forma descontínua. Em campo ocorre, também, uma perda na atividade biológica do resíduo do produto com o passar do tempo e em algum momento pode haver uma concentração do resíduo que seja suficiente para controlar os indivíduos suscetíveis, mas não para controlar os resistentes, ocorrendo assim a evolução da resistência.

\section{Monitoramento da suscetibilidade de populações de Brevipalpus phoenicis aos acaricidas óxido de fenbutatin e cihexatin}

Óxido de Fenbutatin: As porcentagens de sobrevivência das diversas populações de $B$. phoenicis à concentração diagnóstica de $180 \mathrm{mg} \mathrm{L}^{-1}$ de óxido de fenbutatin se encontra na Figura 2. Todas as populações avaliadas mostraram-se altamente suscetíveis, não diferindo estatisticamente da população $S$ (testemunha) $(F=1,04 ;$ g.l. $=19,140 ; P>0,05)$. A sobrevivência variou de $0,12 \%$ a $2,33 \%$, sendo que a população $S$ apresentou uma sobrevivência de $0,28 \%$. A população Fischer-8, que recebeu quatro pulverizações com acaricidas organoestânicos no período de 1995-1999 apresentou uma sobrevivência de apenas $0,75 \%$. A população Fischer-1, que não sofreu pressão seletiva com acaricidas organoestânicos no período de 19951999, apresentou sobrevivência de 0,37\%.
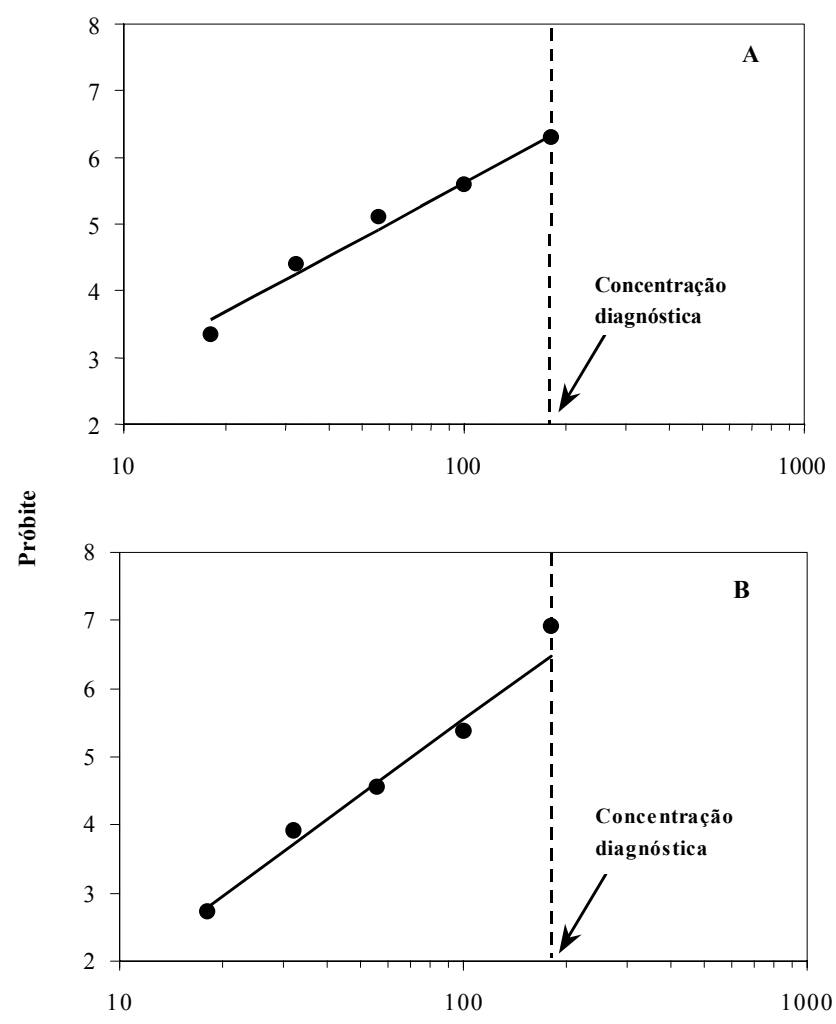

mg de ingrediente ativo $L^{-1}$ de água destilada

Figura 1 - Linha básica de suscetibilidade da população (S) de Brevipalpus phoenicis ao acaricida óxido de fenbutatin (A) e cihexatin (B) por meio de um bioensaio residual (as setas indicam a concentração diagnóstica de 180 $\left.\mathrm{mg} \mathrm{L}^{-1}\right)$.

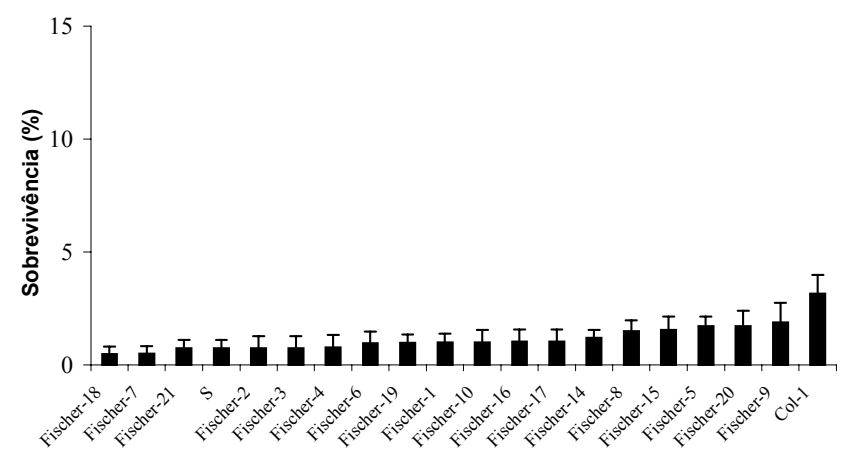

Figura 2 - Médias de sobrevivência ( \pm EPM) em diferentes populações de Brevipalpus phoenicis submetidas à concentração diagnóstica de $180 \mathrm{mg}$ de óxido de fenbutatin $\mathrm{L}^{-1}$ de água destilada. 
Cihexatin: As porcentagens de sobrevivência das diversas populações de $B$. phoenicis à concentração diagnóstica de $180 \mathrm{mg} \mathrm{L}^{-1}$ de cihexatin se encontra na Figura 3. Neste monitoramento, as diversas populações de $B$. phoenicis mostraram-se, também, bastante suscetíveis ao cihexatin. Apenas a resposta da população Col-1 foi significativamente maior que a população $S(F=2,63$; g.l. $=19,80 ; P<0,05)$, que apresentou uma sobrevivência média de $10,72 \%$ na concentração diagnóstica. A população $S$ apresentou uma sobrevivência média de $1,92 \%$. A população Fischer-1 não foi monitorada uma vez que foi perdida devido a problemas na criação. Neste caso, a população Fischer-2 recebeu a menor intensidade de uso de acaricidas organoestânicos (apenas uma pulverização em 1995), apresentou uma sobrevivência de $4,05 \%$. A população Fischer-8 (4 pulverizações de organoestânicos) apresentou uma sobrevivência de $6,01 \%$.

No presente estudo foi caracterizado a concentração diagnóstica de $180 \mathrm{mg} \mathrm{L}^{-1}$ de óxido de fenbutatin e cihexatin que poderá ser utilizada em programas futuros de monitoramento da suscetibilidade de populações de $B$. phoenicis a esses acaricidas

Apesar do intenso uso de acaricidas organoestânicos para o controle de B. phoenicis em citros no Estado de São Paulo, as populações desse ácaro ainda apresentam um alta suscetibilidade a óxido de fenbutatin e cihexatin. Análise do histórico de aplicações de acaricidas no período de 1995 a 1999 nas populações de $B$. phoenicis avaliadas no presente estudo (TABELA 1) revelam que nos pomares que receberam - maior número de aplicações de acaricidas organoestânicos, o seu uso foi no máximo de uma aplicação por ano. Talvez, esse regime de uso de acaricidas organoestânicos (uma aplicação a cada 12 meses) tenha possibilitado o restabelecimento anual da suscetibilidade de populações de $B$. phoenicis a esse grupo de acaricidas. Nessas condições, a rotação de acaricidas organoestânicos com outros grupos de acaricidas seria altamente desejável (Omoto, 1995b; 1998).

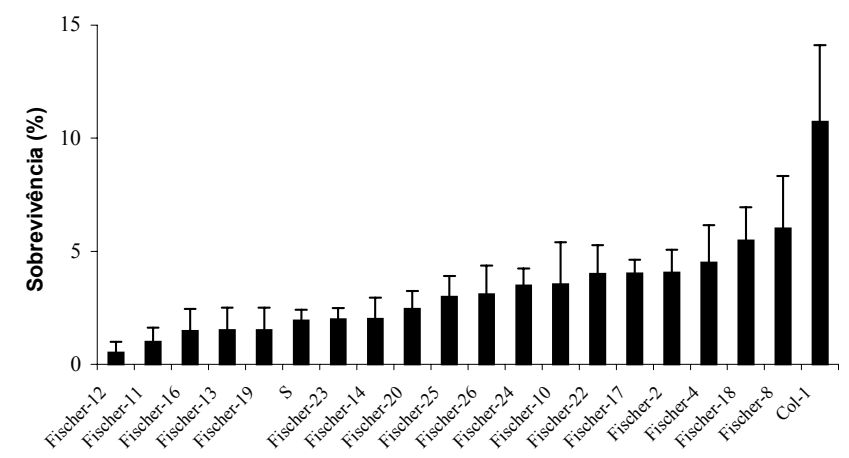

Figura 3 - Médias de sobrevivência ( \pm EPM) em diferentes populações de Brevipalpus phoenicis submetidas à concentração diagnóstica de $180 \mathrm{mg}$ de cihexatin $\mathrm{L}^{-1}$ de água destilada.
Os acaricidas organoestânicos atuam de modo distinto dos demais pesticidas utilizados em citros; ou seja, na respiração celular através da inibição da fosforilação oxidativa (Corbett et al., 1986; Ware, 1989). Outros grupos de acaricidas que atuam na mitocôndria (por ex.: pirroles, pirazóis, propargite, enxofre, etc.) agem em processos bioquímicos diferentes. Sendo assim, a pressão de seleção com acaricidas organoestânicos em $B$. phoenicis tem sido apenas com os produtos utilizados diretamente para o seu controle. Por outro lado, o uso de dicofol no controle de $B$. phoenicis pode ser afetado pelo uso de bromopropilato para o controle de Phyllocoptruta oleivora num mesmo pomar devido à resistência cruzada (Alves, 1999). Acaricidas piretróides ou nor-pirétricos (por ex.: bifentrina, fenpropatrina, acrinatrina, etc.) podem sofrer a pressão de seleção através do uso de inseticidas piretróides no controle de outras pragas em citros. Portanto, a definição de uma estratégia de uso de produtos químicos em um determinado pomar deve ser realizado através da análise conjunta de todo complexo de pragas que ocorrem em um determinado pomar.

\section{AGRADECIMENTOS}

À Fundação de Amparo à Pesquisa do Estado de São Paulo - FAPESP pelo financiamento do projeto e concessão da bolsa de estudos; ao Fundo Paulista de Defesa da Citricultura - Fundecitrus e à Fischer Agropecuária S.A. pelo apoio ao projeto.

\section{REFERÊNCIAS BIBLIOGRÁFICAS}

ALVES, E.B. Manejo da resistência do ácaro da leprose Brevipalpus phoenicis (Geijskes, 1939) (Aacari: Tenuipalpidae) ao acaricida dicofol. Piracicaba, 1999. 91 p. Dissertação (Mestrado) - Escola Superior de Agricultura "Luiz de Queiroz", Universidade de São Paulo.

CHIAVEGATO, L.G. Biologia do ácaro Brevipalpus phoenicis em citros. Pesquisa Agropecuária Brasileira, v.21, p.813816, 1986.

CHIAVEGATO, L.G. A leprose dos citros no Estado de São Paulo. Laranja, v.8, p.7-19, 1987.

CORBETT, J.R.; WRIGHT, K.; BAILLIE, A.C. The biochemical mode of actions of pesticides. London: Academic Press, 1986. cap.1, p.1-49: Pesticides interfering with respiration.

EDGE, V.E.; JAMES, D.G. Organo-tin resistance in Tetranychus urticae (Acari: Tetranychidae) in Australia. Journal of Economic Entomology, v.79, p.1477-1483, 1986.

FFRENH-CONSTANT, R.H.; ROUSH, R.T. Resistance detection and documentation: the relative roles of pesticide and biochemical assays. In: ROUSH, R.T.; TABASHNIK, B.E. (Ed.) Pesticide resistance in arthropods. New York: Chapman \& Hall, 1990. p.4-48.

GOODWIN, S.; HERRON, G.; GOUGH, N.; WELLHAM, T.; ROPHAIL, J.; PARKER, R. Relationship between insecticideacaricide resistance and field control in Tetranychus urticae (Acari: Tetranychidae) infesting roses. Journal of Economic Entomology, v.88, p.1106-1112, 1995. 
HERRON, G.A.; EDGE, V.E.; ROPHAIL, J. The influence of fenbutatin oxide use on organotin resistance in two-spotted mite Tetranychus urticae Koch (Acari: Tetranychidae). Experimental \& Applied Acarology, v.18, p.753-755, 1994.

HOSKINS, W.M.; GORDON, H.T. Arthropod resistance to chemicals. Annual Review fo Entomology, v.1, p.89-122, 1956.

HOSKINS, W.M.; CRAIG, R. Uses of bioassay in entomology. Annual Review Entomology, v.7, p.437-464, 1962.

HOY, M.A.; CONLEY, J.; ROBINSON, W. Cyhexatin and fenbutatin-oxide resistance in pacific spider mite (Acari: Tetranychidae): stability and mode of inheritance. Journal of Economic of Entomology, v.81, p.57-64, 1988.

HOYT, S.C.; WESTIGARD, P.H.; CROFT, B.A. Cyhexatin resistance in Oregon populations of Tetranychus urticae Koch (Acarina: Tetranychidae). Journal of Economic Entomology, v.78, p.656-659, 1985.

JACOBSON, R.J.; CROFT, P.; FENLON, J. Response to fenbutatin oxide in populations of Tetranychus urticae Koch (Acari: Tetranychidae) in UK protect crops. Crop Protection, v.18, p.47-52, 1999.

KEENA, M.A.; GRANETT, J. Cyhexatin and propargite resistance in populations of spider mites (Acari: Tetranychidae) from California almonds. Journal of Economic Entomology, v.80, p.560-564, 1987.

KITAJIMA, E.W.; LOVISOLO, O.; COLARICCIO, A.; CHAGAS, C.M.; ROSSETI, V. Vírus causador da leprose dos citros. In: OLIVEIRA, A.A.L.; DONADIO, L.C. (Ed.) Leprose dos citros. Jaboticabal: FUNEP, 1995. p.19-24.

LEORA SOFTWARE. POLO-PC: a user's guide to Probit or Logit analysis. Berkeley: LeOra software, 1987.

NAKANO, O.; SANCHES, G.A.; ISHIDA, A.K. Redução na infestação do ácaro da leprose Brevipalpus phoenicis (Geijskes, 1939) em citros através do controle da verrugose. Laranja, v.8, p.19-33, 1987.

NAKANO, O. Rotatividade de ingredientes ativos em citros. In: OLIVEIRA, C.A.L.; DONADIO, L.C. (Ed.) Leprose dos citros. Jaboticabal: FUNEP, 1995. p.189-194.

NATIONAL RESEARCH COUNCIL. Pesticide resistance: strategies and tactics for management. Washington: National Academic Press, 1986. p.271-278: Prediction or resistance risk assessment. In: National Research Council

OLIVEIRA, C.A.L. Flutuação populacional e medidas de controle do ácaro da leprose Brevipalpus phoenicis (Geijskes, 1939) em citros. Laranja, v.7, p.1-31, 1986.

OMOTO, C. Manejo da resistência de ácaros e insetos aos produtos químicos na citricultura. Laranja, v.16, p.187-208, 1995a.
OMOTO, C. Resistência de Brevipalpus phoenicis (Acari: Tenuipalpidae) aos produtos químicos na citricultura. In: OLIVEIRA, C.A.L.; DONADIO, L. C. (Ed.) Leprose dos citros. Jaboticabal: FUNEP, 1995b. p.179-188.

OMOTO, C. Acaricide resistance management of leprosis mite (Brevipalpus phoenicis) in Brazilian citrus. Pesticide Science, V.52, p.189-198, 1998.

PREE, D.J.; WAGNER, H.W. Occurrence of cyhexatin and dicofol resistance in European red mite, Panonychus ulmi (Koch) (Acari: Tetranychidae) in Southern Ontario. The Canadian Entomologist, v.119, p.287-290, 1987.

ROUSH, R.T.; MILLER, GL. Considerations for design of insecticide resistance monitoring programs. Journal of Economic Entomology, v.79, p.293-298, 1986.

SALVO FILHO, A. Notas sobre o tratamento fitossanitário em citros. Laranja, v.18, p.155-163, 1997.

TIAN, T.; CARDWELL, E.G.; GRANETT, J. Resistance of Tetranychus urticae (Acari: Tetranychidae) to cyhexatin and fenbutatin oxide in California pears. Journal of Economic Entomology, v.85, p.2088-2095, 1992.

VESTERGAARD, S.; GILLESPIE, A.T.; BUT, T.M.; SCHREITER, G.; EILENBERG, J. Pathogenicity of the hyphomycete fungi Verticillum lecanii and Metarhizium anisophiae to the Western flower trips, Frankliniella occidentalis. Biocontrol Science and Technology, v.5, p.185-192, 1995.

WARE, G.W. The pesticide book. Fresno: Thompson Publications, 1989. cap.17, p.153-166: Modes of action for insecticides.

WELTY, C.; REISSIG, W.H.; DENNEHY, T.J.; WEIRES, R.W. Cyhexatin resistance in New York populations of European red mite (Acari: Tetranychidae). Journal of Economic Entomology, v.80, p.230-236, 1987.

WELTY, C.; REISSIG, W.H.; DENNEHY, T.J.; WEIRES, R.W. Comparison of residual bioassay methods and criteria for assessing mortality of cyhexatin-resistant European red mite (Acari: Tetranychidae). Journal of Economic Entomology, v.81, p.442-448, 1988.

YAMAMOTO, P.T.; PINTO, A.S.; PAIVA, P.E.B.; GRAVENA, S. Seletividade de acaricidas a inimigos naturais em citros. In: OLIVEIRA, C.A.L.; DONADIO, L.C. (Ed.) Leprose dos citros. Jaboticabal: FUNEP, 1995. p.159-170.

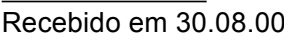

